\title{
Más allá de la corriente dominante: El valor heurístico de la Psicosis Cicloide
}

\author{
F.M.M.A. van der Heijden, MD* \\ S. Tuinier, $\mathrm{MD}, \mathrm{PhD}$ * \\ L. Pepplinkhuizen, MD, PhD** \\ H.M. van Praag, MD, PhD*** \\ W.M.A. Verhoeven, MD, PhD",** \\ * Instituto de Psiquiatría Vincent van Gogh, \\ Venray \\ ** Universidad Erasmus, Facultad de \\ Medicina y Ciencias Médicas, Rotterdam \\ *** Asesor científico del Instituto del \\ Cerebro y del Comportamiento, Universidad \\ de Maastricht, Maastricht \\ HOLANDA
}

\begin{abstract}
RESUMEN - Los intentos para diagnosticar los trastornos psiquiátricos son tan antiguos como la propia Medicina y han ido siempre paralelos con su desarrollo científico. Los conceptos diagnósticos de la Psiquiatría actual están profundamente enraizados en la tradición europea del siglo XIX en la que existe una línea de desarrollo alemana y francesa junto con otra neuropsiquiátrica. A pesar de los intentos rigurosos por elaborar diagnósticos médicos acertados, las dificultades fundamentales relacionadas con la selección de signos y síntomas relevantes y la posterior validación de las entidades sindrómicas, todavía persisten. Uno de los ejemplos más interesantes es la psicosis cicloide que trasciende a las fronteras estrictas de las actuales taxonomías e introduce signos y síntomas alternantes relacionados con experiencias subjetivas y con trastornos de motilidad distintos de la catatonia.

Aunque la práctica actual de categorización descriptiva puede ser útil a efectos de comunicación, nosotros propugnamos la aplicación de un modelo multiniveles tanto horizontal como vertical en el proceso de diagnóstico psiquiátrico.
\end{abstract}

\section{Introducción histórica}

Desde que existen documentos escritos podemos encontrar ya referencias a sínto- mas y enfermedades psiquiátricas. Como reseñaba Jeste et al. (1985), existen descripciones de enfermedades psiquiátricas en la antigua Mesopotamia y en La India, las cuales describen varios tipos de demencia que 
se asemejan a los trastornos bipolares afectivos y a la esquizofrenia. Van Praag (1988) reflexionó con elegancia sobre la melancolía de Job en la Biblia. En la época grecorromana las enfermedades psiquiátricas ya se clasificaban de acuerdo con la sintomatología (melancolía y manía), la etiología (frenitis o demencia acompañada de fiebre) o la evolución (crónica, similar a la esquizofrenia). Estos trastornos fueron descritos por Hipócrates y Celso (1853). Este último fue el primero que utilizó el término delirium. Tanto el delirium como la frenitis y la letargia fueron clasificados como trastornos mentales agudos y separados de las enfermedades con una evolución crónica (Lipowski 1991).

En la época medieval varias fuentes dan fe de la conciencia que se tenía de la enfermedad mental y del cuidado de los enfermos mentales. Wilmer y Scammon (1954) encontraron documentos del siglo XII en los que se hablaba de casos de histeria,neurastenia, epilepsia y trastornos psicóticos. Los trastornos psicóticos podían ser curados milagrosamente, lo que significa que, o bien no se tomaba nota de la evolución crónica de los mismos o que se preocupaba en estos casos de trastornos psicóticos agudos y pasajeros. Muy famoso en esa época fue Bartholomaeus Anglicus, que publicó su enciclopedia "Sobre la propiedad de las cosas" en la que se mencionaban varios trastornos psiquiátricos.

Aunque las historias convencionales de la Psiquiatría describen al periodo medieval y moderno como dominado por las ideas demonológicas sobre la enfermedad mental, siendo el tratamiento de los dementes cruel e inhumano (Neugebauer 1978), cuando se utilizan otras fuentes, emerge entonces una imagen radicalmente diferente. A partir de los documentos legales se hace evidente que se establecían distinciones entre retraso mental y locura en sus formas tanto crónica como recidivante. Además, las considera- ciones etiológicas implicaban principalmente a factores biológicos como daños e infecciones del cerebro (Berger 1999, Von Bingen traducido del latín) así como a causas psicológicas (Beek 1969, Texier 1991).

Una escuela de medicina muy influyente fue la de Galeno (129-198 d.c.) la cual puso de relieve cómo el Sistema Nervioso era el centro de las sensaciones, del movimiento voluntario y de todos los procesos del pensamiento. Él creía, además que los trastornos de la mente se originaban en el cerebro y eran provocados por un desequilibrio de los cuatro humores:el calor de la sangre originado por el corazón, la frialdad de las mucosas proveniente del cerebro, la sequedad de la bilis amarilla del hígado y la humedad de la bilis negra del bazo. (Harkins 1963, Godderis 1988).

$\mathrm{Al}$ principio de la época moderna se puso de manifiesto el valor de la observación clínica, aunque interpretada en términos galénicos. Diethelm (1971) revisó 1.100 disertaciones médicas desde la Edad Media hasta 1750 que trataban los aspectos psicopatológicos, en particular de la melancolía, la manía, la catalepsia, la histeria, prácticamente de todas hipocondriasis y también de los trastornos cerebrales orgánicos. De estos datos se puede concluir que la Psiquiatría era ya una rama de la Medicina y que el tratamiento incluía tanto métodos biológicos como psicológicos. La misma imagen emerge de los archivos de la jurisdicción de Florencia (Magherini y Biotti 1998). Felix Platter (1536-1614) describió minuciosamente varias enfermedades psiquiátricas y formuló una taxonomía que incluía trastornos afectivos, psicosis funcionales, trastornos delirantes y estados de delirio (Diethelm 1965). Puesto que no se hicieron distinciones entre las distintas formas de trastornos psicóticos, podemos catalogarlo como una forma temprana de la denominada Einheitpsychose. 
La taxonomía psiquiátrica se inició en el siglo XIX. Antes de esa época hubo varios intentos de clasificación realizados, por ejemplo, por Thomas Sydenham (16241689), Thomas Willis (1621-1675) y Georg Ernst Stahl (1660-1734). Los tres describieron varios estados patológicos de la mente. El siglo XVIII puede considerarse como el de los albores de la Psiquiatría científica, ya que durante él se publicaron las primeras visiones científicas de la locura basadas en premisas nosológicas (especialmente relativas a la psicosis) (Palha y Esteves 1997). Después de Sydenham, el término enfermedad se convirtió en un término compuesto que describía los rasgos comunes de una variedad de casos individuales y la nosología en una rama de la Medicina preocupada por su definición. Posteriormente, William Cullen (1710-1790) desarrolló una clasificación natural de trastornos mentales tomando los mecanismos etiopatogénicos como criterio principal de clasificación (Berrios y Bear 1995, Pichot 1994). Como resultado de los cambios socioculturales habidos en el cuidado de los pacientes mentalmente enfermos, los médicos iniciaron la descripción de la psicopatología individual. Esta orientación nosográfica fue particularmente defendida por Pinel (1745-1826) y Esquirol (1772-1840), que pueden considerarse los fundadores de la Psiquiatría clínica. Ambos recalcaron la importancia del estudio profundo de los síntomas y el abandono de cualquier teoría coherente sobre las causas y los mecanismos de la locura, así como sobre la disociación de la neurosis de Cullen desde la alienación mental (Pichot 1994, Klosterkötter 1999). Pichot (1986) enfatizaba correctamente la similitud entre la orientación de Pinel y Esquirol y el DSM con respecto a un punto de vista teórico. Después de esto, impulsados por el desarrollo de la Neurología, pero dificultados por la comprensión limitada de las funciones cerebra- les, los trastornos psicóticos quedaron asociados con alteraciones no específicas del sistema nervioso central. Este enfoque finalmente culminó con la Einheitpsychose de Griesinger, que implica que diferentes complejos de síntomas psicológicos son sólo fases de la misma evolución de la enfermedad. Griesinger (1817-1860) postulaba que las enfermedades mentales eran el resultado de los diferentes niveles de energía nerviosa o fisiológica. Según este concepto, la melancolía era el trastorno fundamental que podía derivar en demencia por la vía de alteraciones en el pensamiento y en la voluntad (Griesinger 1861). La caída de la Ein heitpsychose fue anunciada por el propio Griesinger y completada por el muy influyente nosólogo Kahlbaum. Desde ese momento se pueden trazar en la Psiquiatría europea dos líneas de desarrollo, una francesa y otra alemana, la Psiquiatría francesa era más descriptiva, mientras que la Psiquiatría alemana representaba la situación opuesta (Pichot 1992).

Resumiendo, desde las épocas más antiguas se ha informado sobre los síntomas de las enfermedades mentales, se han realizado intentos por definir trastornos específicos y se han postulado especulaciones acerca de su sustrato, situado principalmente en el cerebro. Los desarrollos en la Psiquiatría siempre han ido paralelos a los de la Medicina en general y, como en el caso de la Neurología, se han hecho intentos por encontrar ubicaciones cerebrales para los síntomas y trastornos psicopatológicos. Especialmente estimulantes fueron los descubrimientos de Paul Broca (1824-1880) y de Carl Wernicke (1848-1905), que presentaron evidencia anatómica sobre las funciones cerebrales localizadas. Uno de los ejemplos más atractivos sobre la interdependencia existente entre desarrollos médicos y diagnósticos psiquiátricos, fue el de la clásica histeria de Charcot que desapareció en un 
periodo relativamente corto, como resultado de los avances habidos en la instrumentación médica del diagnóstico (Micale 1993).

\section{Fundamentos de las tendencias contemporáneas en el diagnóstico psiquiátrico}

Desde la segunda mitad del siglo XIX, todos los desarrollos habidos pueden caracterizarse por las respuestas a la pregunta: ¿cuáles son los signos y síntomas relevantes?,¿cómo deberían agruparse estos en síndromes coherentes? y ¿qué ideas pueden adelantarse con respecto a la etiología y a la patogénesis? Cuatro tendencias principales emergen como resultado de las distintas respuestas a estas preguntas.

La primera fue el enfoque estrictamente nosológico, como adelantaron Kahlbaum (1828-1899) y Kraepelin (1856-1926), que finalmente dio como resultado el DSM. La segunda aplica un método más descriptivo y empezó con Pinel (1745-1824) y Magnan (1835-1916), llevando finalmente al sistema de diagnóstico francés utilizado actualmente (Pull et al. 1987 a, b). Este incluye una serie de trastornos psicóticos de los que la bouffée délirante se incluye en el ICD-10 (WHO 1992). La tercera tendencia tuvo su origen en Wernicke (1848-1905), Meynert (1833-1882) y Bonhoeffer (1865-1948). La aplicación del método de la Botánica a la Psiquiatría por parte de los nosólogos por una parte y la influencia de las teorías psicológicas y sociológicas por otra, así como la diferenciación profesional entre la Neurología y la Psiquiatría, llevaron a un declive de la posición ventajosa que tenía la Neuropsiquiatría. El descubrimiento de los fármacos psicotrópicos y el cada vez mayor conocimiento Neurocientífico ha resucitado la Neuropsiquiatría bajo una forma novedosa en la que la funcionalidad de los sistemas cerebrales se halla conectada con los procesos psicológicos y emocionales.

\section{Enfoque nosológico}

Kahlbaum fue el primero que postuló las denominadas entidades nosológicas, planteándose como hipótesis la estrecha correspondencia entre síntomas clínicos, evolución y resultado, teniendo en cuenta la patología cerebral y la etiología. Él describió estadosevolución-entidades clínicas, tales como la parafrenia, la catatonia, la hebefrenia y la ciclotimia (Jablensky 1999; Braunig y Kruger 2000). Las unidades de clasificación eran entidades de enfermedad prototípicas después de los intentos de Virchov por clasificar a los trastornos físicos de forma categorial. Posteriormente Kraepelin sintetizó las observaciones y trabajos de otros y organizó su propio Tratado nosológico (Kraepelin 1896). Como reseñó con elegancia Klosterkötter (1999) y evaluó de forma crítica Van Praag (1993), la nosología de Kraepelin incluía enfermedades endógenas, exógenas y psicogénicas, que fueron clasificadas de acuerdo con etiologías somáticas o neurológicas, con su pronóstico, con la edad de aparición o con la existencia de síntomas prototípicos. Desde Kraepelin se han realizado muchos intentos por formular clasificaciones diagnósticas alternativas de las psicosis, sin embargo su esquema nosológico no fue cuestionado seriamente, ni siquiera por Schneider (18871967). Este último recalcó que el diagnóstico de esquizofrenia podía establecerse incluso en ausencia de los síntomas de primer rango y que la Psiquiatría en general trata con síndromes psicopatológicos, que son útiles o no, en lugar de que sean fundamentalmente acertados o equivocados (Hoenig 1983).

Aunque es algo generalmente aceptado que no existe una prueba simple o norma de proce- 
dimiento que establezca la validez de un concepto de enfermedad o de una clasificación diagnóstica en Psiquiatría (Kendell 1975a), los rasgos esenciales del intento de Kraepelin siguen estando presentes en los sistemas de clasificación actuales (Jablensky 1997). Según Kendell (1975b, 1982) una gran mayoría de los pacientes no se ajustan a las metódicas descripciones estereotipadas que encontramos en las taxonomías modernas, necesitando asignarles más o menos arbitrariamente a cualquier síndrome al que se ajusten más aproximadamente. Comentarios críticos comparables los ha hecho Van Praag, que analizó repetidamente los defectos del enfoque nosológico (Van Praag 1982a,b, 1993,1997).

\section{Enfoque descriptivo}

A finales del siglo XIX, la Psiquiatría francesa estaba dominada por el concepto de degeneración propuesto por Morel (18091873). En su forma más cruda, la noción de degeneración implicaba que podía aparecer en una generación una anormalidad leve, en la siguiente generación le seguiría otra algo más grave, como la locura, y en la tercera la imbecilidad completa. Esta idea, que en nuestros días podría traducirse como formas hereditarias de enfermedad mental, siguiendo el principio genético de la anticipación, no implicaba un principio de categorización sino que pretendía separar las enfermedades mentales congénitas de las formas no hereditarias. El punto central, no obstante, se hallaba en la descripción detallada de la psicopatología (Fairet 1878-1879, Legrain 1886). El representante más destacado de la Psiquiatría francesa en aquella época fue Magnan, que distinguió entre trastornos degenerativos $\mathrm{y}$ aquellos otros que no estaban relacionados con la degeneración. Los primeros incluían la démence précoce de Morel, los estados delirantes agudos denominados bouffées déliran - tes des dégénerés y los estados delirantes crónicos sin una evolución sistemática. Los últimos incluían a los estados delirantes crónicos denominados délires de persécution à évolu tion systématique, así como otros estados afectivos y trastornos orgánicos (Pichot 1984, 1992, 1995). En su Manual, Magnan (1897) describió una serie de psicosis agudas que denominó arrebatos delirantes (bouffée déli rantes o délires polymorphes), locura transitoria o persistente y locura parcial o general, los cuales surgían sin ninguna causa aparente o después de un incidente poco importante.

A lo largo de los años posteriores, la teoría de la degeneración se fue abandonando gradualmente, pero el enfoque descriptivo originado en Esquirol persistió, especialmente en el concepto de bouffées délirantes des dégénerés. Este trastorno psicótico se caracteriza por una aparición repentina y un estado delirante pasajero, así como por un contenido y expresión polimorfos, junto con un estado alterado de la conciencia (Ey et al. 1960, Ey 1973).

\section{El enfoque Wernicke-Kleist- Leonhard}

En contraposición a la clasificación dicotómica de Kraepelin, Wernicke empezó a desarrollar una taxonomía de las psicosis que incluía muchos grupos, basados en su propia patofisiología especulativa. Su idea principal era que los distintos estados psicóticos tienen su origen en alteraciones funcionales de distintos sistemas cerebrales. Esto condujo a novedosos síndromes psicopatológicos, tales como la psicosis de motilidad (Kleist 1926, Fünfgeld 1936). Finalmente realizó una subdivisión muy elaborada de todos los trastornos mentales, incluyendo la denominada $p s i$ cosis marginal (Randpsychosen), que fueron posteriormente divididas en tres formas: $p s i$ cosis de motilidad hipercinética-acinética, 
psicosis de confusión excitada-estuporosa y psicosis delirante ansiosa-extática (Perris 1988, Teighmann 1990, Jablensky 1999).

El trabajo de Kleist fue posteriormente modificado y desarrollado por Leonhard, que recalcó acerca de la independencia nosológica de las psicosis cicloides de los trastornos afectivos, así como de los esquizofrénicos y sobre la evolución recurrente no seguida de ningún defecto. Según Leonhard $(1961,1995)$ la psicosis de ansiedadeuforia se caracteriza por una alteración básica en el humor, que varía entre la ansiedad y el éxtasis; la psicosis de confusión se caracteriza a su vez por un pensamiento desordenado y por la perplejidad, y la psico sis de motilidad por alteraciones en la actividad psicomotriz. Posteriormente se hizo evidente que no siempre era factible una distinción clara de los tres subtipos de psi cosis cicloide y que la mezcla de sintomatología era más la norma que la excepción (Perris 1974, 1988). Por ello, la psicosis cicloide se convirtió en el común denominador del tercer grupo de psicosis funcionales.

\section{Paradigma neuropsiquiátrico}

Como se ha esbozado antes, las teorías acerca de la conexión entre los trastornos mentales y las alteraciones funcionales y/o estructurales del Sistema Nervioso Central han estado presentes a lo largo de la historia de la Medicina. Junto con el desarrollo de la Neurología, la Neuropsiquiatría académica creció mucho en Europa durante la segunda mitad del siglo XIX. Como resultado de la institucionalización de los enfermos mentales, las diferencias en el enfoque con respecto a estos pacientes eran evidentes entre los profesionales de los manicomios y los neuropsiquiatras académicos. La rápida aceptación de las explicaciones psicológicas para las alteraciones mentales desafió a la Neuropsiquiatría académica en la primera mitad del siglo XX y la Psiquiatría se separó de la Neurología y de la Neuropsiquiatría (Benson 1996, Pichot 1997). La Neuropsiquiatría recibió un nuevo ímpetu científico con el descubrimiento de los fármacos psicotrópicos en los años cincuenta y el posterior desarrollo de la Psicofarmacología y la Psiquiatría Biológica, así como por el rápido aumento del conocimiento en los campos de la Neuroimagen, la Neuropatología, la Neuroquímica y la Neurobiología molecular. Esto ha llevado en las dos últimas décadas a un enorme progreso en el conocimiento detallado de las funciones cerebrales, que al mismo tiempo venía a denunciar las deficiencias del diagnóstico psiquiátrico.

Recientemente ha surgido una conciencia creciente de que los fármacos psicotrópicos, tales como los antidepresivos, no tienen ninguna acción diagnóstica específica, que su desarrollo está todavía conectado con hipótesis psiquiátricas biológicas originadas en los años cincuenta y sesenta y que además las explicaciones extracelulares simples sobre su acción deberían sustituirse por un mejor conocimiento acerca de los mecanismos intracelulares y de trascripción génica (Verhoeven y Tuinier 2001, Holsboer 1999). Además, ni los estudios neuropatológicos ni los biológicos han demostrado la existencia de factores patogenéticos relacionados causalmente con una determinada entidad nosológica, lo que da como resultado la indeterminación del diagnóstico y los denominados problemas de comorbilidad (Van Praag et al, 1990a,b, Tuinier y Verhoeven 1995, Tuinier et al, 1996, Brieger y Marneros 2000). Además, hay una evidencia de peso acerca de que la dicotomía cartesiana entre Psicología y Biología en Psiquiatría ya no es defendible frente a los resultados de otras investigaciones exhaustivas que demuestran el impacto fundamental de factores de desarrollo y psicosociales sobre la función cerebral y en la expresión de los determinantes genéticos (Kraemer y Clarke 1990, Kraemer et al. 1997, 
De Kloet et al. 1996, Kandel 1998, 1999, Post 1992, Kraus 2000). Ese punto de vista ya fue expresado a principios de los años sesenta por Van Praag, que abogaba por una distinción entre etiología (en parte psicológica) y patogénesis y enfatizaba su interdependencia (Van Praag y Leijnse 1964). La patogénesis fue definida como la serie de disfunciones cerebrales subyacentes a un trastorno del comportamiento; la etiología como la serie de factores (biológicos y psicológicos) que desencadenaban las disfunciones cerebrales.

Todos estos desarrollos recientes han dado un nuevo impulso a la Neuropsiquiatría en la que el holismo frente a la localización es sustituido por un mayor interés en los sustratos anatómicos funcionales del comportamiento teniendo en cuenta los trastornos del comportamiento normal y patológico. Se ha hecho evidente que diferentes sistemas cerebrales están implicados en la expresión de los síntomas psiquiátricos, de los cuales dos, han recibido la mayor parte de la atención de la investigación hasta ahora. El primero abarca los circuitos fronto-subcorticales que están, entre otros, bajo el control dopaminérgico hallándose implicados en una amplia variedad de funciones como la motivación, el comportamiento ejecutivo, la cognición y el afecto. El segundo tiene que ver con la integridad funcional del eje hipotalámico-pituitario-adrenal (HPA) y ejerce su papel sobre los comportamientos de adaptación y en los estados psicopatológicos de los que son representantes principales los trastornos de personalidad y del humor, la ansiedad y los trastornos cognitivos (De Kloet 1991, De Kloet et al. 1996, Rupprecht y Holsboer 1999, Ellembroek y Cools 2000). Como consecuencia de ello, la investigación moderna se centra en la conexión entre la disfunción cerebral y esos parámetros psicológicos más sutiles (Saint-Cyr $e t$ al. 1995, Cummings 1993, Cummings y Hegarty 1994), lo que puede llevar en un futuro cercano a novedosos enfoques diagnósticos (Cowan et al. 2000).

Los problemas arriba mencionados en relación con el diagnóstico psiquiátrico como la confusión sindrómica, la rigidez nosológica y la comorbilidad han inducido a Van Praag desde 1965 a abogar por una psicopatología funcional, que constituye el cuarto nivel de un enfoque diagnóstico que incluye la definición nosológica, la tipología sindrómica, la diferenciación de los síntomas y la evaluación de las disfunciones psicológicas subyacentes y que tiene como objetivo, en última instancia, analizar minuciosamente el trastorno psicopatológico en las partes que lo componen. (Van Praag y Leynse 1965; Van Praag 1990, 1993, 1997).

El diagnóstico psiquiátrico de acuerdo con un modelo nosológico puede tener una función comunicativa pero no debe limitar la percepción de los signos y los síntomas que puedan estar relacionados con disfunciones tanto psicológicas como del Sistema Nervioso Central. De acuerdo con el paradigma del cerebro y del comportamiento, que es la base de la Neuropsiquiatría, el proceso diagnóstico en Psiquiatría debería implicar un modelo multinivel tanto horizontal como vertical. El modelo horizontal supone la utilización de un enfoque polidiagnóstico que implica la aplicación de varios sistemas en paralelo para el diagnóstico y clasificación de un grupo único de pacientes con el objetivo último de definir clases sindrómicas con una mayor validez interna de constructo. (McGorry et al. 1992, 1998) La verticalización implica la adopción de un enfoque diagnóstico en tres niveles para los fenómenos psicopatológicos (Van Praag 1993, 1997). Por supuesto, deben evaluarse y registrarse sistemáticamente muchas otras variables, como la discapacidad o los problemas funcionales, la evolución de la enfermedad, las pautas familiares, la patopsi- 
cología y la respuesta a los tratamientos (Lieberman y Rush 1996).

Un ejemplo muy claro sobre los efectos adversos de una categorización rígida es la exclusión de la psicosis cicloide la cual posee una constelación específica de síntomas psicopatológicos.

\section{El concepto de psicosis cicloide}

Según Leonhard (1961, 1995), la existencia de un trastorno mental, en este caso una psicosis endógena, se corrobora por la consistencia de un grupo de síntomas recurrentes, una evolución específica, el efecto del tratamiento y la presencia o ausencia de cargas familiares. Las características principales de la psicosis cicloide son la recuperación total, un inicio subagudo y una sintomatología polimorfa (Pfuhlmann 1998, Sigmund y Mundt 1999). Es interesante señalar como las psicosis que no encajaban en la estricta clasificación dicotómica de Kraepelin, fueron presentadas de forma independiente en las primeras décadas del siglo XX al igual que las formas cíclicas e intermitentes de la demencia precoz (Dunton 1910), las psicosis esquizoafectivas agudas (Kasanin 1933), las bouffées delirantes (Ey et al. 1960, 1967), las psicosis atípicas (Mitsuda 1965, Hatotani 1996) y las psicosis degenerativas (Schröder 1920). Todos estos trastornos psicóticos tienen en común una aparición repentina, una sintomatología polimorfa, una recuperación completa y un buen pronóstico. Debemos recalcar que la descripción original de Kasanin de trastorno esqui zoafectivo es fundamentalmente diferente del trastorno esquizoafectivo incluido en las taxonomías actuales, que se basa principalmente en la presencia de síntomas esquizofrénicos (Marneros y Tsuang 1986, May 1984).
El concepto de psicosis cicloide fue elaborado con detalle por Perris (1974), que la describió así. La psicosis cicloide se caracteriza en el nivel sintomático por oscilaciones del humor, confusión, trastornos de la motilidad, ansiedad generalizada, síntomas distónicopsicóticos del humor y ocasionalmente éxtasis, mostrando todos ellos una expresión fluctuante de gravedad. Las observaciones longitudinales ofrecen un episodio único o bien recurrente con remisión completa. Distintos episodios, no obstante ello, pueden no coincidir con la expresión del conjunto completo de síntomas. Un posterior conocimiento de estos criterios llevó a confeccionar un listado formal de éstos, publicado originalmente por Perris y Brockington (1981) y que incluye tanto los síntomas contados como los observados, así como algunos factores no sintomatológicos, tales como son la aparición de los mismos y su duración. Posteriormente estos criterios fueron adaptados por la Asociación Mundial de Psiquiatría (Berner et al. 1992) y se incluyeron en un formato más descriptivo en la clasificación ICD-10.

El concepto diagnóstico de psicosis cicloi de fue validado de diferentes maneras. Pri mero, el análisis factorial reveló un conjunto específico de síntomas de los cuales cinco tienen un mayor valor predictivo: perplejidad, desorientación, fluctuaciones de motilidad fásicas, felicidad-éxtasis y ansiedadpánico (Jönson et al. 1991). Brockington y colaboradores, demostraron que la psicosis cicloide, tal como se define por una lista que incluye diversos items, entre otros, el comienzo y la evolución, los síntomas y signos, los antecedentes y la respuesta al tratamiento, cumple los criterios como para constituirse en una entidad sindrómica independiente (Brockington y Roper 1990, Brockington et al. 1991). Además, aplicando un enfoque polidiagnóstico, McGorry et al. (1992) demostraron una tasa de concordancia diagnóstica moderadamente alta entre la psicosis esqui - 
zoafectiva de Kasanin, la bouffée delirante y la manía esquizoafectiva RDC. No existía concordancia diagnóstica con el trastorno esquizoafectivo DSM-III-R ni con la psicosis atípica. Finalmente se encontró una fiabilidad de intervaloración elevada para la psico sis cicloide (Pfuhlmann et al. 1997). Segun $d o$, en cuanto a la validez del pronóstico, se ha demostrado repetidamente que este tipo de psicosis tiene un buen pronóstico sin que se produzcan estados defectuales afectivos o del comportamiento, a pesar de su reagudización, mostrando un perfil sintomático diferente del que posee el trastorno bipolar afectivo, la esquizofrenia o los trastornos psicoafectivos (Cutting et al. 1978, Brockington et al. 1982a, b, Perris, 1988, May 1990, Cutting 1990, Torgalsbøen 1999, Zaudig 1990, Pfuhlman et al. 2000). Además, Beckmann et al. (1990) en un estudio prospectivo mostraron resultados clínicos muy favorables en pacientes con psicosis cicloide que, dentro del marco del DSM, fueron clasificados como trastornos esquizofrénicos o esquizoafectivos. Muy interesantes son las observaciones de Franzek y Beckmann (1991, 1992) quienes, mediante la aplicación de la clasificación de esquizofrenia de Leonhard, señalaron cómo pueden delinearse subgrupos homogéneos con respecto a la evolución, resultado y carga familiar. Tercero, existe alguna evidencia acerca de un impacto, sólo marginal, de los factores genéticos, especialmente en comparación con los trastornos afectivos bipolares (Franzek y Beckmann 1998a, b). Además,otros datos de estudios epidemiológicos han revelado una incidencia de un año para las psicosis cicloi des del 0,04\%, que es la mitad de la incidencia de la esquizofrenia, con una tasa más elevada para las mujeres (Lindvall et al. 1986, 1990, 1993). Esto último puede explicarse por el riesgo existente entre las mujeres de la psicosis cicloide posparto (Shöpf y Rust 1994, Pfuhlmann et al. 2000). Finalmente, las investigaciones neurobiológicas revelaron que la sintomatología covariaba significativamente con el flujo sanguíneo hemisférico (Warkentin et al. 1992), que la amplitud del P-300 auditivo no difería del de los controles (Strik et al. 1993) y que ninguno de los pacientes con psicosis cicloide presentaba ventrículos agrandados como se ha observado mediante la tecnología CT-scan (Höffler et al. 1997). Estos descubrimientos, no obstante, pudieran estar faltos de especificidad.

Con respecto a la patogénesis de la psico sis cicloide, disponemos de evidencias de que en un subgrupo con dispercepciones importantes se dan asociadas, anormalidades en el metabolismo de los aminoácidos (Pepplikhuizen 1983, Bruinvels y Pepplikhuizen 1984. Rijn-van den Meijdenberg et al. 1994, Fekkes y Pepplikhuizen 1997). Recientemente se informó sobre los efectos positivos del estradiol en el tratamiento de un pequeño grupo de psicosis posparto (Ahokas et al. 2000) lo que puede sugerir un papel patogenético de los esteroides en el caso de la psicosis cicloide, aunque también se encontraron niveles de estradiol en plasma inferiores, en pacientes del sexo femenino con episodios psicóticos agudos heterogéneos (Huber et al. 2001).

Con respecto al tratamiento farmacológico, varios autores han sugerido que los antipsicóticos son efectivos sólo en la fase aguda pudiendo inducir a largo plazo un estado de déficit pseudoesquizofrénico (Perris 1991, Leonhard 1995). Los estudios retrospectivos revelaron que la evolución de la enfermedad no se ve influida en absoluto o apenas, por los antipsicóticos, lo que también sucede en psicosis emocionales parecidas a la esquizofrenia y en las esquizoafectivas (Labhardt 1963, Johnstone et al. 1978, Pepplinkhuizen 1983). Los estabilizadores del humor, como el litio, son las combinaciones de primera elección (Perris 1978 1991) pero hasta ahora, 
no obstante ello no se han presentado estudios de tratamientos controlados.

\section{Comentarios finales}

Los intentos de diagnosticar los trastornos psiquiátricos son tan antiguos como la propia Medicina y siempre han ido en paralelo a su desarrollo científico. Como se ha comentado, la adhesión estricta a un sistema nosológico, implica una estrechez de miras de forma que los fenómenos no incluidos se consideran inexistentes o irrelevantes. De hecho, la validez de dichas entidades nosológicas no se basa en datos empíricos sino en la intuición de médicos que trabajaron hace muchos años y que fueron codificados por comités de expertos (Van Praag et al. 1990, Brockington et al. 1991, Mindhall et al. 1992). Nosotros, por tanto, abogamos por abandonar la conceptualización unitaria del diagnóstico, con su adhesión a un sistema diagnóstico determinado, a expensas de la observación y del empirismo. En base a los criterios clásicos que definen a una enfermedad médica: cuadro clínico, historia natural, etiología, patogénesis y respuesta al tratamiento (Guze 1978), determinados trastornos psiquiátricos, como el trastorno obsesi vo compulsivo, el delirium surgido después de dejar el alcohol, las demencias, el trastor no de déficit motivacional y la posible psico sis cicloide, pueden considerarse como entidades nosológicas. En el caso de otros,como las psicosis funcionales, los trastornos del humor y de ansiedad, así como los trastor nos neuróticos y de personalidad, se requiere una orientación dimensional (Van Praag 1993, Knoll et al. 1998, Seivewright et al. 1998).

Varios autores han recalcado la importancia que tienen los parámetros subjetivos junto a los cambios observables somáticos o bien del comportamiento, como factores a incluir en la clasificación (Van Praag 1992, Pichot 1994). Con respecto a la psicosis cicloide, Barcia (1994) analizó la sintomatología en referencia a la experiencia específica del paciente. Generalmente los pacientes se hallan en un estado de confusión incoherente, ausentes y distanciados, a veces con una apariencia de perplejidad y de ansiedad, pero especialmente con una expresión vaga que se refleja en su mirada, como si estuvieran en un sueño. En ocasiones tienen dificultades para determinar si están despiertos o soñando, es decir se hallan en lo que denominamos estado onírico. Los síntomas se experimentan además como si fuesen externos a ellos. El concepto de psicosis cicloide heurísticamente posee interés, puesto que trasciende los límites estrictos de las taxonomías actuales e introduce signos y síntomas alternativos relacionados con experiencias subjetivas, como la perplejidad, el desconcierto emocional y los sentimientos extáticos así como los trastornos de la motilidad distintos a los propios de la catatonia. Además, la evolución en el tiempo de la sintomatología tiene lugar con rápidas fluctuaciones. Tal y como lo analizó Van Praag (1976, 1993), las psicosis funcionales incluyendo la esquizofrenia, no son en absoluto entidades homogéneas. Él, por tanto, sugiere estudiar grupos sintomatológicamente más homogéneos con el fin de identificar sus bases patogénicas. En el caso de la psicosis cicloide, los parámetros funcionales más relevantes podrían ser la fluctuación rápida, la actitud del sujeto hacia los síntomas, concretamente, la perplejidad y la alteración de la motilidad.

Por tanto, una de las cuestiones fundamentales no resuelta en la Psiquiatría es si los signos y los síntomas son sólo indicativos de "Anzeichen" o son una expresión de "Ausdrucke" un proceso patogénico; diferencia importante señalada por Edmund Husserl 
(1992) en su famoso trabajo "Logische Untersuchungen". Esto queda bien ilustrado en el campo de la Psiquiatría en el caso del Retraso Mental, en el que diferentes trastornos genéticos y sus correspondientes anormalidades cerebrales conducen a una serie de síntomas psiquiátricos no específicos a partir de distintas categorías de diagnóstico. Por otra parte, los síndromes con una etiología genética específica pueden estar asociados con típicos fenotipos psicológicos y psicopatológicos del comportamiento que tienen una expresión muy variable (Verhoeven et al. 2000, Verhoeven y Tuinier 2001).

En conclusión, a pesar de los intentos rigurosos por construir diagnósticos médicos fidedignos, las dificultades fundamentales relacionadas con la selección de signos y síntomas relevantes y la posterior validación de entidades sindrómicas, todavía persisten. El concepto de psicosis cicloide puede determinarse todavía más, demostrando su validez sindrómica, la relevancia de las dimensiones psicopatológicas específicas y por último definiendo la conexión entre disfunciones psicológicas específicas y su sustentación neurobiológica. En la investigación neurobiológica, debe seguirse un enfoque neuropsiquiátrico, en el que la psicopatología funcional fuese el "Leitmotiv".

\section{Reconocimientos}

Esta reseña es la primera parte del proyecto de investigación "Psicosis atípicas", aprobado por el Comité Ético Médico de la Universidad Erasmus de Rotterdam y respaldado económicamente por una beca ilimitada de Eli Lilly, Países Bajos B.V. La primera parte del título es una paráfrasis de una de las publicaciones de Van Praag.

\section{Bibliografía}

BARCIA, D. The clinical evaluation of cycloid psychoses. In: Pichot P, Rein W (eds): The clinical approach in psychiatry. Collection Les Empêcheurs de Penser en Rond, 305-321, 1994.

BARTHOLOMAEUS, A. On the properties of things: John Trevisa's translation (from the Latin) of Bartholomaeus Anglicus "De proprieta-tibis rerum"; a critical text. Oxford Clarendon Press, 1975-1988.

BERRIOS, G.E.,BEER, D. Unitary Psychosis Concept. In:GE Berrios \& R Porter (eds.), A history of clinical psy chiatry. The origin and history of psychiatric disorders. Athlone, London, 1995.

BROCKINGTON, I.F., PERRIS, C., KENDELL, R.E., HILLIER, E. The course and outcome of cycloid psychosis. Psychol Med, 12, 97-105, 1982a.

CELSUS. The first four books of De Re Medica. Translated by Steggall J. London Churchill, 1853.

COWAN, W.M., HARTER, D.H., KANDEL, E.R. The emergence of modern neuroscience: some implications for neurology and psychiatry. Ann Rev Neurosci, 23:343-391,2000.

CUTTING, J.C. Relationship between cycloid psychosis and typical affective psychosis. Psychopathology, 23: 212-219, 1990.

DE KLOET, E.R., ROTS, N.Y., COOLS, A.R. Braincorticosteroid hormone dialogue: slow and persistent. Cell Mol Neurobiol, 1996; 16: 345-356.

DIETHELM, O. Medical dissertations of psychiatric interest. Printed before 1750. Karger Basel, 1971.

EY, H.,BERNARD, P.,BRISSET, C.H. Psychoses délirantes aigues. In: Manuel de Psychiatrie. Masson \& Cie Paris 1960: 244-274.

FEKKES, D., PEPPLINKHUIZEN, L. Amino acid studies in transient acute polymorphic psychosis. Amino Acids' 12: 107-117, 1997.

FRANZEK, E., BECKMANN, H. Different genetic background of schizophrenia spectrum psychoses: A twin study. Am J Psychiatry, 155: 76-83, 1998a.

GUZE, S.B. Nature of psychiatric illness: Why psychiatry is a branch of medicine. Compr Psychiatry, 19: 295307, 1978.

HOLSBOER, F. The rationale for corticotropin-releasing hormone receptor (CRH-R) antagonists to treat depression and anxiety. J Psychiatr Res, 33: 181-214, 1999.

HUSSERL, E. Logische Untersuchungen. I: Prolegom ena zur reinen Logik. Meiner Hamburg, 1992. 
JABLENSKY, A. The conflict of the nosologists : Views on schizophrenia and manic-depressive illness in the early part of the 20 $0^{\text {th }}$ century. Schizophr Res, 39: 95-100, 1999.

JÖNSSON, S.A.T.,JONSSON, H.,NYMAN, G.E. The concept of cycloid psychosis: The discriminatory power of symptoms. Acta Psychiatr Scand, 84: 22-25, 1991.

KANDEL, E.R. A new intellectual framework for psychiatry. Am J Psychiatry, 155: 457-469, 1998.

KANDEL,E.R. Biology and the future of psychoanalysis:A new intellectual framework for psychiatry revisited. Am J Psychiatry, 156: 505-524, 1999.

KLEIST, K. Über cycloide Degenerationspsychosen, besonders Verwirrtheits- und Motilitätspsychosen. Zentral blatt für die gesamte Neurologie und Psychiatrie, 44:655656, 1926.

KLOSTERKÖTTER, J. Psychiatrische Klassifikation. Fortschr Neurol Psychiat, 67: 558-573, 1999.

KRAEMER, G.W.,SCHMIDT, D.E.,EBERT, H.M. The behavioural neurobiology of self-injurious behaviour in rhesus monkeys. Current concepts and relations to impulsive behaviour in humans. Ann NY Acad Sci, 836:12-38,1997.

LEONHARD, K. Aufteiling der endogenen Psychosen und ihre differenzierte Ätiologie. Thieme Verlag Stuttgart, 1995.

LINDVALL, M.,AXELSSON, R., ÖHMAN, R. Incidence of cycloid psychosis. Eur Arch Psychiatry Clin Neu rosci, 242: 197-202, 1993.

MAGHERINI, G.,BIOTTI, V. Madness in Florence in the $14^{\text {th }}-18^{\text {th }}$ centuries. Judicial inquiry and medical diagnosis, care and custody. Int J Law Psychiatry, 21:355-368,1998.

McGORRY, P.D., SINGH, B.S., CONNELL, S., McKENZIE, D., VAN RIEL,R.J.,COPOLOV, D.L. Diagnostic concordance in functional psychosis revisited: A study of inter-relationships between alternative concepts of psychotic disorder. Psychol Med, 22: 367-378, 1992.

MICALE, M.S. On the "disappearance" of hysteria. A study in the clinical deconstruction of a diagnosis. Isis, 84: 496-526, 1993.

MINDHAM R.H.S., SCADDING, J.G., CAWLEY, R.H. Diagnoses are not diseases. Br J Psychiatry, 161: 686-691, 1992.

NEUGEBAUER, R. Treatment of the mentally ill in medieval and early modern England:A reappraisal. J Hist Behav Sci, 14: 158-1691, 1978.

PERRIS, C. Cycloid psychotic disorders ("Marginal Psychoses"). In: Seva A (ed) The European handbook of psychiatry and mental health. Prensas Universitarias de Zaragoza, 1000-1009, 1991.
PFUHLMANN, B. Das Konzept der zyloiden Psychosen. Fortschr Neurol Psychiat, 66: 1-9, 1998.

PICHOT, P. The French approach to psychiatric classification. Br J Psychiat, 144: 113-118, 1984.

PICHOT, P. Nosological developments in European psychiatry and psychopharmacology. Pharmacopsychia try, 19: 23-25, 1986.

SIGMUND, D., MUNDT, C. The cycloid type and its differentiation from core schizophrenia:A phenomenological approach. Compr Psychiatry, 40: 4-18, 1999.

TEXIER, P. La rémission au XIVème siècle. Genèse et developpement. Thèse (doctorat d'état inédit) Limoges, 1991.

TUINIER, S., VERHOEVEN, W.M.A. Dimensional classification and behavioral pharmacology of personality disorders; a review and hypothesis. Eur Neuropsychophar macol, 5: 35-146, 1995.

VAN PRAAG, HM. About the impossible concept of schizophrenia. Compr Psychiatry, 17: 481-497, 1976.

VAN PRAAG, H.M. Job's agony: A biblical evocation of bereavement and grief. Judaism, 37: 173-187, 1988.

VAN PRAAG, H.M. "Make-believes" in psychiatry or the perils of progress. Clinical \& Experimental Psychiatry Monograph N. ${ }^{\circ}$ 7. Brunner/Mazel New York, 1993.

VAN PRAAG, H.M., LEIJNSE, B. Neubewertung des Syndroms. Skizze einer funktionellen Pathologie. Psychiat Neurol Neurochir, 68: 50-66, 1965.

VAN PRAAG, H.M., ASNIS, G.M., KAHN, R.S., BROWN, S.I., KORN, M., HARKAVY FRIEDMAN, J.M.,WETZLER, S. Nosological tunnel vision in biological psychiatry. A plea for a functional psychopathology, Ann NY Acad Sci, 600:501-510, 1990a.

VERHOEVEN, W.M.A., TUINIER, S., CURFS, L.M.G. Prader-Willi Psychiatric Syndrome and Velo-Cardio-Facial Psychiatric Syndrome. Genet Couns, 11: 205213,2000 .

VERHOEVEN, W.M.A., TUINIER, S. Two steps forward, one step back; paradigmatic changes in psychiatry. $J$ Neural Transm, 2001, in press.

WILMER, H.A., SCAMMON, R.E. Neuropsychiatric patients reported cured at St. Bartholomew's hospital in the twelfth century. J Nerv Ment Dis, 119: 1-22, 1954.

Dirección para correspondencia:

Prof. Dr. W.M.A. Verhoeven

Vincent van Gogh Institute for Psychiatry

P.O. Box 5

5800 AA Venray

HOLANDA 